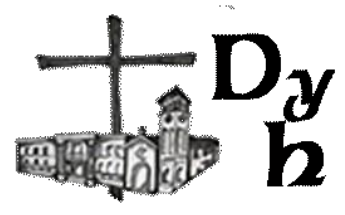

El debate en torno a la mímesis en la República de Platón y en la Poética de Aristóteles Julián Lanusse

Dios y el hombre, vol. 4, n. 1, e055, 2020

ISSN 2618-2858 - https://doi.org/10.24215/26182858e055 https://revistas.unlp.edu.ar/DyH/index Cátedra libre de pensamiento cristiano - UNLP Seminario Mayor San José La Plata, Buenos Aires, Argentina

\title{
EL DEBATE EN TORNO A LA MÍMESIS EN LA REPÚBLICA DE PLATÓN Y EN LA POÉTICA DE ARISTÓTELES
}

\author{
The Discussion about the Mimesis \\ in the Republic by Plat and in the Poetics by Aristotle \\ Julián Lanusse \\ julilanusse19@gmail.com \\ Universidad Nacional de La Plata - La Plata - Argentina
}

\section{Resumen}

En el presente artículo se busca analizar la concepción de mímesis tanto en la filosofía platónica, especialmente a partir de La República, como a lo largo de la Poética de Aristóteles. Considerando esto, se busca confrontar ambos planteos para rescatar ciertos aspectos de cada uno y proponer una visión propia que retoma la concepción de arte y belleza de la tradición escolástica, especialmente la lectura que realiza el filósofo Jacques Maritain que supone una especie de síntesis a la concepción de los autores de la antigua Grecia.

Palabras claves: Aristóteles; Escolástica; Mímesis; Platón

\begin{abstract}
This article tries to analyze the conception of mimesis both in Platonic philosophy, especially studying the Republic and Aristotle's Poetics. On this basis, it intends to confront both approaches to rescue certain aspects of each one and to propose an original vision that takes into account the conception of art and beauty of the scholastic tradition, especially the reading made by the philosopher Jacques Maritain, which is a kind of synthesis of the conception of the two authors of ancient Greece.
\end{abstract}

Keywords: Aristotle; Mimesis; Plato; Scholasticism 


\section{Introducción}

Siguiendo al filósofo ingles Alfred North Whitehead $(1929,63)$, podemos afirmar que "la historia de la filosofía occidental no es más que una serie de notas de pie de página a Platón". Este punto resulta relevante para el presente artículo no solamente debido a que buscamos analizar las concepciones que tienen Platón y Aristóteles sobre la imitación, llamada mímesis en griego, sino que también buscamos comprenderlas teniendo en cuenta el contexto en el que escriben y la línea teórica que siguen los planteos que vienen desarrollando.

En el caso de Platón observamos, como se analizará en el primer apartado, que desarrolla una filosofía dirigida críticamente hacia la creciente democracia ateniense y la importancia que esta le concedió a los sofistas, pensadores que serán llamados "comerciantes del saber" y a los cuales se les negará el nombre de filósofos. Por otro lado, observamos como el Estagirita plantea ciertas cuestiones de su filosofía estableciendo una clara y sólida distancia respecto a su maestro Platón. Estos aspectos adquieren importancia en el caso de los escritores antiguos ya que permiten llegar a comprenderlos de una forma más cercana a su pensamiento, algo que Julián Marias $(1968,16)$ llama restaurar los textos "como se hace con un viejo pergamino" en contraposición a lo que el mismo filósofo denomina proyectar sobre el texto nuestra situación propia, lo que termina provocando una mala interpretación de la filosofía antigua y nos puede llevar a caer en un anacronismo.

A su vez, hay otro aspecto por el cual es interesante analizar los planteos de las filosofías desarrolladas por Platón y Aristóteles: estos han sido los primeros filósofos de los cuales se han conservado casi íntegramente sus escritos, en palabras de Marias decimos que ambos autores "nos han legado, excepcionalmente, su obra, si no completa, al menos en sus porciones esenciales" (Marias, 1968, 15). Esto último nos permite ver cómo la filosofía occidental se constituye como una respuesta a la filosofía platónica y, por lo tanto, a la aristotélica. A esto hacemos alusión cuando hablamos de la superior importancia que adquieren estos filósofos a la hora de contemplar el conjunto de la historia filosófica occidental.

Considerando esto, a continuación se presentan dos apartados que dan cuenta de la concepción de la mímesis tanto en Platón como en Aristóteles para luego concluir en la última sección rescatando los aportes de cada uno y 
añadiendo una perspectiva propia en cuanto a esta temática, rescatando ciertos aspectos de la filosofía escolástica que sintetiza Jacques Maritain.

\section{La mímesis platónica}

Para comenzar a entender la concepción de mímesis que elabora Platón a lo largo de la República, es necesario entender los planteos de base que lo llevan a hablar sobre la imitación. En este sentido, creo que es conveniente centrarnos en dos aspectos de la filosofía platónica que conducen al autor a concebir la mímesis de una manera determinada y particular. En primer lugar, es importante entender la visión que Platón tiene del alma debido a que esta va a ser la que condicione todo su posterior desarrollo teórico ${ }^{1} \mathrm{y}$, por otro lado, tener en claro el impacto que tiene esta concepción del alma en la educación y en el gobierno de la polis. Para esto último, es relevante afirmar que Platón escribe en un contexto en donde la democracia ateniense se consolidaba, distanciándose de una Esparta cada vez más inclinada en sus intereses militares, lo que provocó que distintos pensadores, entre ellos los sofistas, lleguen a la polis. A su vez, estos últimos aspectos son necesarios para poder seguir el hilo del desarrollo por parte de Platón debido a que en el fondo de su discurso encontramos una fuerte crítica a la democracia y a la educación ateniense ${ }^{2}$.

Con respecto al primer punto mencionado, Platón piensa en un alma inmaterial y eterna que posee una estructura tripartita: una parte racional cuyas virtudes son la sabiduría y la prudencia, otra irascible caracterizada por el valor y la fortaleza y, por último, una concupiscible en donde se encuentran la templanza y la moderación. Considerando esto, el filósofo desarrolla su intelectualismo ético ${ }^{3}$ y afirma que cada parte del alma tiene que vivir de acuerdo a su virtud y que, a su vez, la parte racional tiene que encontrarse subordinando a las demás partes ${ }^{4}$. A pesar de esto último, Platón sostiene que en cada persona predomina una determinada parte del alma $y$, debido a esto,

\footnotetext{
${ }^{1}$ Como se menciona infra, Platón llegará a sus conclusiones definitivas en el libro $\mathrm{X}$ de República, tomando como base, pues, todo su anterior despliegue teórico.

2 Esto, además, entendido como una reacción platónica ante la injusta condena de su maestro Sócrates. De aquí también comprendemos la dura crítica que hace el filósofo en contra de los sofistas.

${ }^{3}$ Esta postura también la encontramos defendida por el mismo Sócrates.

${ }^{4}$ Esto es ilustrado por Platón con la analogía del alma con la yunta alada y el auriga en Fedro 246a-249c.
} 
los estamentos de la ciudad tendrían que conformarse por los productores, que son aquellos en los que domina la parte concupiscible, los guerreros, en los cuales hay una relevancia de la parte irascible, y por los filósofos en los que predomina la parte racional. Esta visión, conducirá a Platón a colocar en el centro de su República Ideal la educación, tanto del cuerpo (gimnasia) como del alma (música), la cual debe estar basada en la estructura tripartita de esta última. Todo este desarrollo va a llevar a que Platón, en el libro III de La República, escriba sobre la forma en la cual se tiene que dar la educación musical y cuáles son los recaudos que hay que tomar para velar por el bien del alma, aspecto que se plantea para preservar a los jóvenes de los poemas que puedan corromper su alma ${ }^{5}$. En este marco, el filósofo griego plantea un destierro de aquellos relatos en los que se muestre a los dioses y héroes faltando a la verdad, engañando o cometiendo actos que van contra el bien y la justicia. El argumento utilizado por Platón es que de lo bueno, en este caso, de las divinidades, no puede provenir el mal, por eso escribe lo siguiente:

Pues no creamos todo eso —seguí- ni dejemos que se diga que Teseo, hijo de Poseidón, y Pirítoo, hijo de Zeus, emprendieron tan tremendos secuestros ni que cualquier otro héroe o hijo de Zeus ha osado jamás cometer atroces y sacrílegos delitos como los que ahora les achacan calumniosamente. Al contrario, obliguemos a los poetas a decir que semejantes hazañas no son obra de los héroes, o bien que éstos no son hijos de los dioses, pero que no sostengan ambas cosas ni intenten persuadir a nuestros jóvenes de que los dioses han engendrado algo malo o de que los héroes no son en ningún aspecto mejores que los hombres. Porque, como hace un rato decíamos, tales manifestaciones son falsas e impías, pues a mi parecer quedó demostrada la imposibilidad de que nada malo provenga de los dioses (La República trad. en 1986, 391d).

Además, Platón sostiene que los relatos apropiados para la educación de los jóvenes no deben contener ninguna desobediencia hacia los superiores, aspecto que va en contra de la templanza ${ }^{6}$, y tampoco ninguna escena en

\footnotetext{
${ }^{5}$ En base a esto, se dice "no sea que por causa de ellos se inclinen nuestros jóvenes a cometer el mal con más facilidad" (La República, 392a).

${ }^{6}$ Cf. República, 390a.
} 
donde se transmite un miedo ante la muerte, ya que esto perjudicaría gravemente a los guardianes ${ }^{7}$.

Luego de haber desarrollado lo relativo a los temas abarcados en los poemas, tanto los que son correctos como aquellos que tienen que ser desterrados, Platón se enfoca en la forma de desarrollarlos ${ }^{8}$. En este punto, el debate se centra en si los poemas tienen que ser recitados bajo la narración simple, la imitación en donde el poeta se asimila a otro en habla y en aspecto ${ }^{9}$, o la mezcla entre ambos sistemas. Precisamente, la discusión se enfoca en esta segunda forma, llamada mímesis por el autor:

Pues lo que yo quería decir era precisamente que resultaba necesario llegar a un acuerdo acerca de si dejaremos que los poetas nos hagan las narraciones imitando o bien les impondremos que imiten unas veces sí, pero otras no -y en ese caso cuándo deberán o no hacerlo-, o, en fin, les prohibiremos en absoluto que imiten. (Ibíd., 394d).

En los puntos siguientes del libro III de La República, Platón establece las bases respecto a su visión sobre la mímesis para luego retomarlo en el libro $X$, aspecto que se abordará más adelante en este artículo. En este sentido, el filósofo griego delimita el campo de la imitación teniendo como base la concepción del alma que deja en evidencia que las personas solamente practicarán bien un oficio que es coherente a la parte predominante de su alma, hecho ejemplificado con el caso de los guardianes ${ }^{10}$. Sin embargo, luego afirma que, si estos han de imitar, tienen que acostumbrarse desde niños a imitar con modelos dignos, magnánimos y valerosos, "pero las acciones innobles no deben ni cometerlas ni emplear su habilidad en remedarlas, como tampoco ninguna otra cosa vergonzosa, no sea que empiecen por imitar y terminen por serlo en realidad." (Ibíd., 395d).

Ahora bien, sin duda, en el libro X de La República Platón establece una dura crítica a la poesía retomando los aspectos abordados en su libro III pero construyendo su argumentación a partir de lo escrito sobre el alma, especialmente en los libros $\mathrm{V}$ y VI. Además, en este punto el autor desarrolla su

\footnotetext{
${ }^{7}$ Cf. República, 386a-387b.

${ }^{8}$ Cf. República, 392d

${ }^{9}$ Cf. República, 393c

${ }^{10} \mathrm{Cf}$. República, 394e
} 
punto de vista sobre la base de la relación entre los poemas y la sociedad lo que provocó una serie de fuertes críticas hacia este autor, especialmente de la mano del romanticismo. Es por esto que lo planteado aquí por Platón adquiere una especial importancia por ser en donde plasma sus conclusiones en cuanto al arte mimético. El argumento platónico está centrado en el ejemplo de la divinidad, el artesano y el pintor, al que va a comparar con el imitador, en torno a la construcción de una cama. En este sentido, y siguiendo con su teoría de las Ideas, Platón distingue que existen tres tipos de camas: la que existe en la naturaleza y es fabricada por la divinidad, la que hace el artesano y la que fabrica el pintor. En este punto, el filósofo enfoca su crítica al decir que el artesano realiza una cama como una copia de la Idea de cama proveniente de la

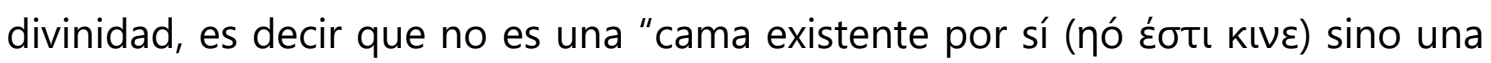

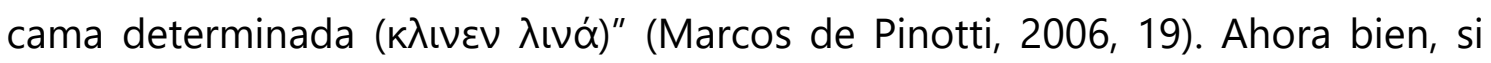
esta copia ya se vuelve un tanto oscura, el pintor, identificado con el imitador, hará algo más alejado todavía. Por tanto, Platón sostiene que el arte imitativo es ontológicamente débil ya que se encuentra a una distancia triple respecto a lo real, es decir que conformaría una mera apariencia debido a ser una copia de una copia. El filósofo afirma que los poemas imitativos "causan estragos en la

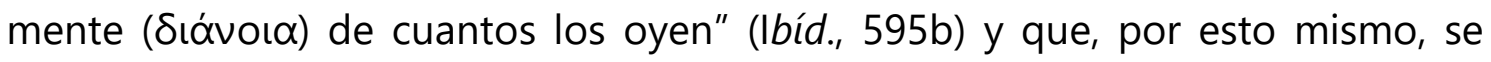
sitúa a Homero como el responsable de esto, "maestro y caudillo de todos los trágicos" (ibíd., 595c), a pesar del cariño y reverencia que desde niño Platón siente por él.

Considerando todo este desarrollo, podemos decir que Platón critica fuertemente el arte imitativo debido a la distancia entre este y la verdad y, por lo tanto, el imitador se presenta como un mago con la aparente capacidad de fabricar no solo los objetos debidos al artesano, sino también "todo cuanto brota de la tierra y produce todos los seres vivos, incluido él mismo, y además de esto, la tierra y el cielo y los dioses y todo lo que hay en el cielo y bajo tierra en el Hades" (Ibíd., 596c). Por todo esto, Platón termina afirmando que "no se ha de admitir de ningún modo en la polis ideal poesía alguna que sea imitativa" (Marcos de Pinotti, 2006,16). 


\section{Mímesis en Aristóteles}

Para abordar la concepción que plantea el Estagirita respecto a esta temática nos centraremos principalmente en su Poética, en la cual plantea que tanto la epopeya como la tragedia, pasando incluso por la comedia y el ditirambo, se constituyen a través de imitaciones (Poética, 1447a). En este sentido, vale la pena agregar que esta obra, que data del año 334 a.C., probablemente no fue concebida personalmente por Aristóteles como una obra escrita, sino que es una recopilación que sus discípulos realizaron de las clases que tomaron con él. Esto se pone de manifiesto en que podemos ver en este escrito digresiones, interrupciones y lagunas a lo largo de sus capítulos, lo que provocó una respuesta de relectura y reescritura especialmente a partir del siglo $X V$. En este texto, el filósofo plantea que, si bien estas artes difieren en los medios a través de los cuales realizan la imitación, principalmente el ritmo, la melodía y el verso, la mímesis entendida como una representación de la realidad y no como una mera imitación de la acción, es común en todas. A su vez, Aristóteles afirma que en algunas artes, como en la tragedia, la comedia y las poesías ditirámbicas y nómicas, tiene lugar una combinación de los medios detallados anteriormente.

Además de las diferencias en cuanto a los medios, las distintas artes se van a distinguir respecto a si las acciones de los agentes que representan son buenas o malas, es decir, que la divergencia radica en si las imitaciones son de personas superiores, inferiores o semejantes a nosotros. Es así que "Polignoto representaba a sus personajes superiores a nosotros, Pausón, peores, y los de Dionisio eran tales como nosotros" (Ibíd., 1448a). A su vez, Aristóteles utiliza esta afirmación como base para distinguir a la tragedia, la cual pinta a los hombres mejores que los del presente como por ejemplo los héroes que afrontan las adversidades que el destino les presentan, y la comedia que los representa peores de lo que son haciendo referencia a una clase específica de faltas, que el filósofo ubica como una especie de lo feo y lo denomina "lo ridículo" (Ibíd., 1449a). En este marco, en el capítulo III de la Poética el Estagirita establecerá una tercera diferencia entre las artes imitativas que radica en la manera en que es representado cada objeto, es decir, si se narra en forma directa en un momento y en otro se asume otro personaje, como lo hace Homero, o si por el contrario los imitadores representan toda la historia. 
Considerando estos planteos, Aristóteles realiza una división de tres partes dentro de la poesía: la lírica que está relacionada con aquellos poemas que eran cantados con el acompañamiento de un instrumento musical de cuerdas llamado lira; la poesía narrativa que es denominada épica ${ }^{11}$; y la dramática en donde se encuentran la tragedia y la comedia con las diferencias mencionadas supra.

Ahora bien, probablemente el clímax de la argumentación aristotélica se encuentre a inicios del capítulo IV cuando el filósofo plantea que "la imitación es natural para el hombre desde la infancia, y esta es una de sus ventajas sobre los animales inferiores, pues él es una de las criaturas más imitadoras del mundo, y aprende desde el comienzo por imitación" (Ibíd. 1448b). Es en este punto cuando el Estagirita coloca a la mímesis dentro de la naturaleza humana y, como si esto fuera poco, afirma que es un aspecto que diferencia al hombre del resto de los animales, por lo que la posibilidad de imitar marca una superioridad ${ }^{12} y$, al ser esta una habilidad connatural al hombre, implica que este se regocije cuando lo hace. Además, Aristóteles va a concederle un lugar fundamental a la mímesis en el origen de la poesía ya que, a partir de las improvisaciones y una serie de mejoramientos graduales, fue la encargada de su creación.

Aunque anteriormente habíamos afirmado que el Estagirita le concede un lugar central a la mímesis en toda la Poesía, en la Poética se centra en la tragedia dejando para una "consideración posterior" el análisis de la comedia (Ibíd., 1449b). Considerando esto, el filósofo retoma lo dicho anteriormente para afirmar que la tragedia implica una representación de una acción digna, es decir, adecuada a la moral de la sociedad, por lo que podemos inferir que toda acción que no sea coherente a esta última no podrá ser representada dentro de la tragedia. Por otro lado, Aristóteles plantea que en la tragedia la acción imitada debe transcurrir a lo largo de un ciclo solar, esto es, en un día. En este punto adquiere importancia el concepto de verosimilitud desarrollado por el filósofo que refiere a algo que, si bien no es real, podría serlo. A su vez, esto otorga las bases para que Aristóteles diferencie entre el poeta y el historiador ya

\footnotetext{
${ }^{11}$ Esta palabra viene del griego ह̌troc, y significa palabra, historia o poema.

12 En base a esto, es interesante colocar aquí algunos estudios recientes, desde un plano psicológico, en donde se ha colocado en un papel importante la tarea de la imitación a la hora de la evolución cultural acumulativa y la posibilidad de reconocer e identificarse con los otros hombres en tanto agentes intencionales y mentales, aspectos que el resto de los animales no comparten debido a la imposibilidad de la imitación (Tomasello, 2007).
} 
que, aunque la obra de Heródoto se trasladara a verso, este último relata lo que sucedió mientras que el poeta habla de lo que podría acontecer o haber acontecido. Por esto mismo, el Estagirita afirma que "la poesía sea más filosófica y de mayor dignidad que la historia" (Ibíd., 1451b). Por último, Aristóteles se refiere al lenguaje cuidado, bueno y elegante que tiene lugar en la tragedia $y$, debido a que sus personajes suelen ser reyes y reinas y héroes y heroínas, los enaltece.

Por otro lado, a partir del capítulo $X$ de Poética, se exponen tres elementos fundamentales que toda tragedia debe poseer para consolidar una

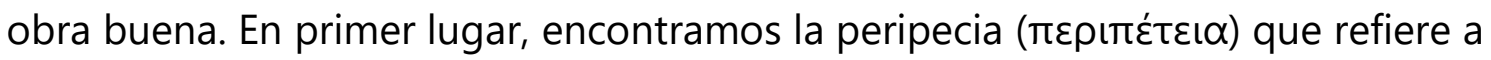
un cambio de suerte en desgracia o viceversa que se da, generalmente, en el protagonista de la obra. En Edipo "el cambio lo produce el mensajero, quien al pretender alegrar a Edipo y eliminar sus temores respecto de su madre, revela el secreto de su nacimiento" (Ibíd., 1452a). En segundo lugar, Aristóteles plantea el reconocimiento (ávapvúpıøı)) cuando se da un paso de la ignorancia al conocimiento, que es en un aspecto simbólico, o cuando un personaje reconoce a otro por señales físicas. Este segundo elemento se ejemplifica también dentro de Edipo cuando pasa a conocer su origen y en el momento en que el pastor lo reconoce a través de las cicatrices de sus pies. Por último, el Estagirita describe la hamartía ( $\dot{\alpha} \mu \alpha \rho \tau i ́ \alpha)$ que es un error trágico que comete el héroe al intentar hacer lo correcto en una situación que, según el filósofo, no puede hacerse. Considerando esto, Aristóteles llama "fábulas simples" a las obras poéticas cuando no contienen estos elementos, y "compuestas cuando" estos ocurren. Luego de este análisis cualitativo de los elementos trágicos, el filósofo toma un punto de vista cuantitativo para referirse a las distintas secciones que posee la tragedia:

El prólogo es todo lo que precede al párodo del coro; un episodio es todo lo que entra entre dos canciones corales completas; el éxodo, todo lo que sigue después de la última canción coral. En las secciones corales del párodo se halla la primera expresión total del coro, y un estásimo es una canción coral sin anapestos o tróqueos. Un commos es un pasaje de lamentos en el que tanto el coro como los actores toman parte (Ibíd., 1452b). 
Por último, Aristóteles aborda la cuestión de que las fábulas deben evitarse y ahonda más en las características propias de la tragedia y el efecto trágico. Principalmente, insistirá en que las formas más bellas de tragedia deben ser fábulas complejas y ordenadas en las que se imiten acciones que provocan temor, que acontece por algo sufrido por hombres semejantes a nosotros, y piedad, que es producida por una desgracia inmerecida; tales son las funciones distintivas de esta clase de imitación. En este sentido, el Estagirita restringe a tres los tipos de fábulas que deben evitarse: en las que un hombre excelente pasa de la felicidad a la desdicha, situación que no es piadosa ni provoca temor sino que nos es odiosa; en las que, a la inversa de la primera, un hombre malo pasa de la desdicha a la felicidad, situación que no presenta requisitos de la tragedia por lo que es la menos trágica que puede presentarse; y por último aquella situación en la que un hombre malo pase de la felicidad a la miseria, debido a que esto despertaría el sentimiento humano sin conducirnos al temor ni a la piedad.

\section{Aristóteles y Platón a la luz de la tradición escolástica}

Teniendo en cuenta los planteos de los filósofos antiguos, considero oportuno introducir la concepción que la escolástica desarrolló a lo largo de la historia, especialmente el despliegue que realiza Maritain en su obra Arte $y$ Escolástica en donde, además de retomar a Tomás de Aquino, hace una síntesis a partir de los planteos platónicos y aristotélicos. De esta manera, es posible rescatar algunos aspectos de los despliegues teóricos de estos autores para proponer una visión estética sobre la cuestión.

En este marco, considero que el problema central dentro del debate respecto a la mímesis radica en la relación entre el arte, la belleza y, en última instancia, el impacto que estas tienen en las personas. Al entender este vínculo, podremos comprender la discusión y proponer una salida superadora. En primer lugar, planteamos que el arte se encuentra en el plano del hacer ya que tiende a realizar una obra (Maritain, 1920, 43). Sin embargo, lo más propio del arte, ya sean los poemas, las fabulas, etc., es la belleza definida como lo que agrada a la vista, id quod visum placet $^{13}$, aspecto que nos permite afirmar que el

\footnotetext{
${ }^{13}$ Maritain (1920, p. 31) es consciente de esto y afirma que esta concepción es propuesta, con "tanta sencillez como sabiduría" por Tomás de Aquino.
} 
verdadero fin del arte, es decir aquello que debe buscar, es la belleza. De esta manera, el acto de hacer una obra bella constituye un fin en sí mismo, un absoluto. Maritain $(1920,44)$ denomina a este tipo de artes como "bellas artes". Ahora bien, considero que las restricciones artísticas que proponen Aristóteles y Platón, las cuales fueron abordadas anteriormente, pueden ser leídas bajo el planteo teórico de Maritain para concluir que el arte es permitido siempre y cuando tenga como fin la belleza. De todos modos, debido a que lo bello pertenece al orden de los trascendentales y supera a los géneros y categorías, afirmamos que la belleza es relativa "a la naturaleza propia y al fin de la cosa, y a las condiciones formales bajo las cuales se la toma" (Maritain, 1920, 39). Por lo tanto, no se debe aplicar los mismos cánones a los poemas que a las fabulas ya que cada tipo de arte se subordina a la belleza en base a lo dicho anteriormente. Evidentemente, esta relatividad perteneciente a la belleza no debe ser entendida similar al relativismo moderno que refiere a las disposiciones del sujeto a la hora de asignar juicios y valores.

Maritain $(1920,31)$ afirma que la belleza es conocida por la inteligencia $y$, por lo tanto, pertenece al mundo inteligible. Este planteo es coherente con la concepción platónica de arte, aunque Maritain también les concede importancia a los sentidos argumentando que estos no solamente sirven a la inteligencia a la hora de conocer la belleza, sino que también gozan en dicho acto. Entonces, la belleza que realmente es connatural al hombre es la que llega a deleitar a la inteligencia a través de los sentidos. En este punto, considero muy oportuna la síntesis que realiza Maritain sin negar que la belleza es un trascendental pero asignándole de todas formas importancia a los sentidos. Por lo tanto, podemos afirmar que las bellas artes son las que tienen el fin del goce mediante la intuición de los sentidos, delectación que va más allá del acto de conocer per se y que implica una alegría que desborda ese acto debido a que el objeto mantiene una proporción con la inteligencia. De esta forma, coincido con Maritain $(1920,33)$ cuando afirma que "la inteligencia goza de lo bello porque en él se reencuentra y se reconoce, y toma contacto con su propia luz". En otras palabras, es un acto que comienza en la persona y la conduce a conocerse a sí mismo a través de un encuentro profundo con su esencia reflejada en aquello que es bello. Teniendo en cuenta esto, podemos coincidir tanto con Aristóteles como con Platón en asignar ciertas restricciones a las artes ya que solamente 
serían buenas las que provoquen este goce de la inteligencia, es decir, aquellas que permitan a la persona encontrarse consigo misma. En este marco, considero que lo central para que un arte sea bueno es, a pesar de los medios que utilice o los aspectos extrínsecos a ella, la dirección del habitus intelectual dirigida a este encuentro con uno mismo y, por lo tanto, enalteciendo la inteligencia.

Por último, si consideramos lo planteado hasta aquí aparece la cuestión de si la mímesis es válida o no en términos artísticos. La posición que tomaremos en este artículo se sustenta en la síntesis realizada en este apartado, la que nos lleva a concebir a la imitación, entendida en tanto representación de lo real, como un medio, es decir, como un signo que hace conocer al alma la belleza del objeto. De esta forma, la mímesis adquiere importancia debido a la función que cumple, pero no constituye como tal el arte. En otras palabras, planteamos que conforma un elemento material del arte utilizado por el artista para "hacer brillar el resplandor de una forma, la luz del ser" (Maritain, 1920, 73). De esta forma, el planteo aristotélico en base a que es la mímesis hace que el hombre se regocije, podríamos contraponerle nuestro pensamiento que refiere a que la inteligencia se deleita no en base a la imitación per se, sino que producto de lo que ella manifiesta. Siguiendo esta lógica, la Pintura imita con colores, la Música con sones y ritmos y la Danza con los movimientos del alma aquel mundo invisible que se agita en nosotros. Son, entonces, un signo de aquello inmerso en nuestra profundidad, lo cual nos trasciende y nos habla de nosotros mismos, de quiénes somos. Buber $(2006,36)$ realiza un aporte interesante al concebir al arte como un lenguaje que emerge del misterio y, a su vez, permite que las personas hablen de él. Considerando esto, afirmamos que la perfección de la obra no radica en el uso correcto de la imitación como reproducción de las cosas sino, de modo contrario, viene de la verdad de la imitación como manifestación de la forma.

\section{Conclusión}

A lo largo del presente artículo, hemos realizado una revisión histórica de la concepción de mímesis en Platón y en Aristóteles, autores que inician el debate y provocan distintos tipos de respuestas en varios autores de la filosofía occidental. En base a esto, observamos que, mientras que en la filosofía platónica la imitación aparece degradando el arte ya que se encuentra 
triplemente alejada ontológicamente de lo real, debido a que constituye una copia de una copia. Por lo tanto, este filósofo afirma que hay que desterrar la mímesis del arte ya que puede corromper el alma y constituye un claro peligro para las personas. Por otro lado, y en contraposición a Platón, el Estagirita plantea que la imitación es connatural al hombre y, debido a esto, para que un arte sea bueno tiene que necesariamente ser imitativa. Como queda en evidencia, mientras que Platón afirma que hay que desterrar al arte mismo ya que consiste esencialmente en una mímesis, Aristóteles plantea que esta debe ser una imitación lo mejor posible.

Dentro de este debate, la posición que tomamos retoma a los filósofos antiguos, pero en una lectura a la luz de la corriente escolástica, acto que, a pesar de implicar un desafío, es necesario para desarrollar un rastreo histórico que permita rescatar los aportes clásicos y complementarlos con la filosofía de nuestro tiempo. Esto ha sido ponderado positivamente por Bergson (1911) quien, en una conferencia en la Universidad de Oxford, valoró con entusiasmo la originalidad y profundidad con la que se estudian allí a los filósofos antiguos al mismo tiempo que se está a la Vanguardia del movimiento filosófico ${ }^{14}$. Considerando esto, concluimos que lo que constituye al arte como tal es el hecho de tener a la belleza como fin, lo que va a producir el deleite de la inteligencia producto de que en ella el ser se encuentra con su esencia. En este sentido, el verdadero acto central del arte es aquel en el cual la forma se vuelve una obra $y$, entonces, lo que permite que al contemplar el arte se revele la forma (Buber, 2006, 19). De esta forma, la mímesis adquiere importancia en la medida que es el medio por el cual se puede manifestar la belleza. En este sentido, considero que es necesario enfocarse en el fin puramente intelectual del arte y no tanto en el habitus manual que, a pesar de permitir una mayor significación, conforma algo extrínseco a las bellas artes. Este punto se ejemplifica a partir del caso de los primitivos que, a pesar de su torpeza, revelaban en su arte de las cavernas una intelectualidad sutil, suceso que no pasa en los museos modernos que, a pesar de los avances científicos y materiales, es decir la habilidad manual, se pierde la belleza del arte, es decir, la expresión de la forma de la cosa.

\footnotetext{
${ }^{14}$ Bergson $(1911,105-6)$ agrega "Estudiemos antes a los antiguos, impregnémonos de su espíritu, y tratemos de hacer, en la medida de nuestras fuerzas, lo que habrían hecho si vivieran con nosotros".
} 


\section{Bibliografía}

Aristóteles, trad. en 1927. Poética

Buber, M. (2006). Yo y Tú. Buenos Aires: Lilmod.

Bergson, H. (1911, mayo). El Pensamiento y lo movimiento. Documento presentado en la Universidad de Oxford.

Marías, J. (1968). La biografía de la filosofía (pp. 10-30). Madrid: Revista de Occidente, S.A.

Marcos de Pinotti, G.E. (2006). La crítica platónica a oradores, poetas y sofistas. Hitos en la Conceptualización de la mímesis. Estudios de Filosofía, 34 (2), 9-28.

Maritain, J. (1920). El arte y la escolástica (pp. 15-83). Buenos Aires: Club de lectores

Platón, trad. en 1986. Fedro

Platón, trad. en 1986. La República

Tomasello, M. (2007). Los orígenes culturales de la cognición humana (pp. 25120). Buenos Aires: Amorrortu.)

Suñol, V (2009). Mimesis en Aristóteles: Reconsideración de su significado y su función en el Corpus Aristotelicum. Tesis de posgrado. Universidad Nacional de La Plata. Facultad de Humanidades y Ciencias de la Educación.

Whitehead, A. (1929). Proceso y realidad (pp. 45-65). Buenos Aires: Losada, S.A. 